\section{УДК 004}

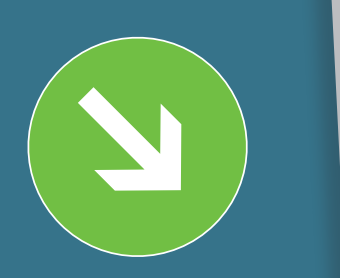

Ключевые слова:

цифровое

предприятие, PLM-

системы, «тяжелые»

PLM-системы, циф-

ровой макет изде-

лия, MDM-системы,

цифровой двойник

промышленного

предприятия

\title{
ЦИФРОВОЕ ПРЕДПРИЯТИЕ: БИТВА СЕТЕВЫХ ПЛАТФОРМ
}

\author{
ОБЗОР. ЧАСТЬ $3 *$
}

\section{Елена ПОКАТАЕВА}

Цифровое производство - это понятие, которое сегодня включает, с одной стороны, все классические этапы жизненного цикла изделия: управление требованиями, проектирование, производство, техническую эксплуатацию, утилизацию. С другой стороны, оно охватывает все сопутствующие процессы: управление проектами и ресурсами (РМ), планирование производства, организацию взаимодействия с заказчиками и партнерами (CRM), обслуживание производственного оборудования и ремонты (ТОиР), работу склада и т.д. Результатом становится пересмотр видов ПО, имеющих критически важное значение для цифровизации предприятия.

\section{PLМ: ЗЕРКАЛО ПРОБЛЕМ ПЕРЕХОДНОГО В0ЗРАСТА ЦИФРОВОГО ПРЕДПРИЯТИЯ}

Показательны результаты последнего исследования Forrester Research систем PLM для дискретных производств: позиции традиционных мировых лидеров, таких как PTC, Dassault Systèmes и Siemens, стремятся захватить софтверные лидеры - SAP и Oracle (рис. 1).

На форуме PDT 2017 года эксперты отметили ключевую проблему PLM-отрасли - устаревание. «Устаревание в ИТ и PLM означает, что решения и программы становятся слишком дорогостоящими в эксплуатации. Или у вас имеются устаревшие программы, но вы хотите использовать преимущества новых перспективных решений», поясняет Хакан Карден, организатор форума PDT. По оценкам аналитиков CIMdata, промышленным предприятиям приходится вкладывать в решение этой проблемы все больше усилий и ресурсов. Например, авиапром - одна из самых передовых отраслей в смысле цифровизации производственной деятельности - сегодня остро ощущает проблемы устаревания PLM. B арсенале PLM и бизнес-ПО компании Boeing около 2500 программных систем и приложений, с которыми рабо-

*Начало см.: СТАНКОИНСТРУМЕНТ, 2018, № 2.

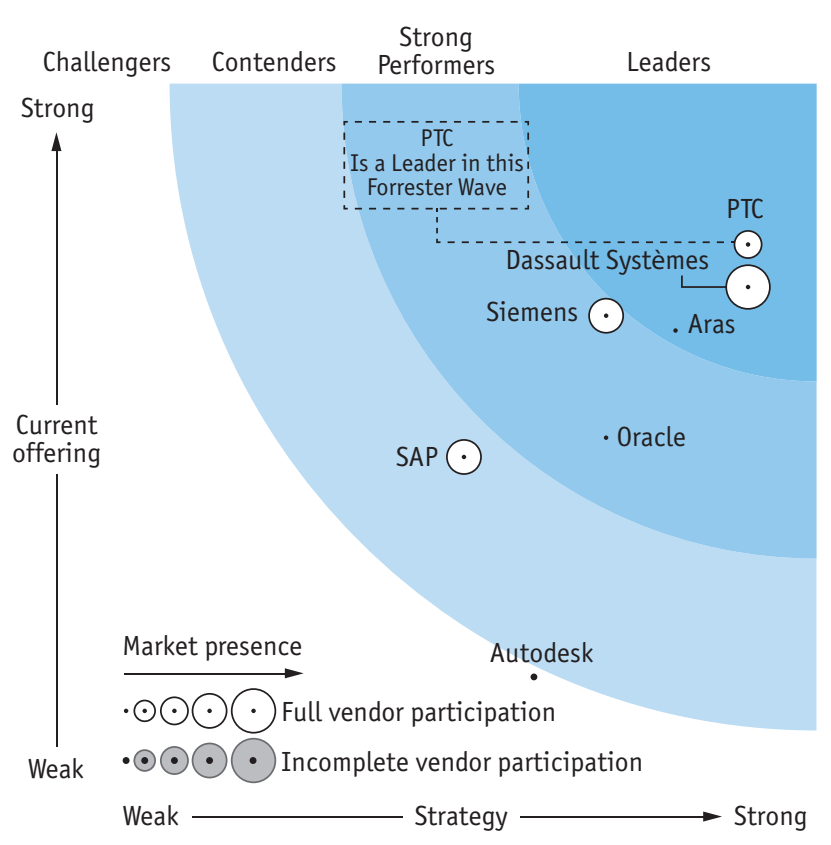

Puc. 1. Глобальные лидеры в сфере PLM

Источник: www.forrester.com

тают примерно 40 тыс. инженеров, использующих 200 стандартов. «Для нас является проблемой кастомизация и проприетарные данные, используемые внутри систем. Это приводит к тому, что 
встраивание в нашу информационную систему новых технологий и возможностей становится весьма трудной интеграционной задачей», - рассказывает Брайан Чизи, директор по средствам интеграции бизнеса в подразделении коммерческих самолетов компании Boeing.

Очевидно, что простая замена одного набора ПО на другое в данной ситуации невозможна. Более реалистичен эволюционный сценарий: тщательно разработанный поэтапный план замены и инвестиции в переподготовку специалистов. В этом, отмечает Олег Шиловицкий, международный PLMэксперт, кроется главный конфликт ПО PLM попытка усовершенствовать организационные процессы, навязывая пользователям изменения: «Это очень трудно сделать. Ситуация дополнительно осложняется тем, что бизнес-интересы вендоров значительно влияют на их подходы к разработке инженерного ПО».

Еще на одну проблему указывает Григорий Абаев, руководитель отдела технической поддержки «Би Питрон»: «Внедрение PLM-проектов по определению требует междисциплинарного подхода». С ним согласен Алексей Боровков, проректор по перспективным проектам СПбПу: «Высокотехнологичное инженерное программное обеспечение нацелено на решение мультидисциплинарных задач, которые так или иначе присутствуют в большинстве различных областей машиностроения».

Методологические вопросы построения современных PLM-решений - предмет обсуждений на различных международных конференциях. «В результате, в мировой практике сформировался определенный подход к созданию полномасштабных PLM-комплексов, - рассказывает Игорь Кочан, директор по маркетингу компании «топ Системы». - Его суть - формирование единого вертикального платформенного решения, на базе которого в дальнейшем строятся все приложения предметной области. Только такой подход позволит избежать многократной конвертации и дублирования данных, необходимости решения задач синхронизации приложений, согласования механизмов безопасности и многих других проблем, с которыми сталкиваются все пользователи, пытающиеся строить PLM-решение не на основе единой платформы, а из набора отдельных приложений, «закрывающих» отдельные направления».

\section{ВРЕМЯ ПЛАТФОРМЕННЫХ РLМ}

Наиболее известные и масштабные платформенные решения глобального уровня разработаны в компаниях Dassault Systèmes и Siemens PLM Software.

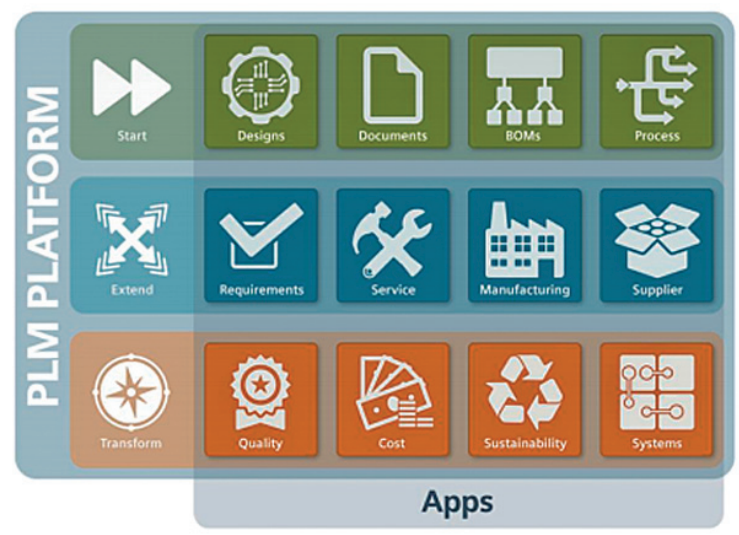

Puc. 2. Ключевая идея архитектуры Siemens Teamcenter - отделение платформы от приложений

ПО Siemens Teamcenter включает платформу и портфель приложений, ориентированных на решение определенного спектра задач. Платформа - это ядро системы, которое включает набор основных базовых компонент и выполняет роль системной шины для развертывания различных приложений (рис. 2).

Teamcenter дает возможность управлять различными типами данных, описывающих изделие на всех стадиях его жизненного цикла: 2Dи 3D-данными, созданными в различных системах проектирования (включая САПР микроэлектроники), графическими и текстовыми документами в любых форматах, наборами атрибутов, объединенных в электронные карточки, и другими видами представления информации. ПО Teamcenter работает на основе единой базы данных об изделии и процессах. При этом обеспечивается совместная коллективная работа конструкторов над единым электронным макетом изделия и параллельная работа над изделием конструкторских и технологических служб.

«Платформа 3DEXPERIENCE, - как рассказывает Алексей Рыжов, генеральный директор Dassault Systèmes в России и СНГ, - это «операционная система цифрового предприятия». Подобно OC компьютера, платформа 3DEXPERIENCE может объединить все области, все этапы производственного процесса, предложить различные инструменты и решение разных задач» (рис. 3). Всего, по оценке Алексея Рыжова, реализовано более 500 прикладных ИТ-решений, предназначенных для определенных профессиональных ролей в разных отраслях с функционалом кастомизации.

Часть приложений тесно интегрирована с CAEрешениями. Например, для обслуживания процесса получения физического прототипа на 3D-принтере используется ПО моделирования того объекта, который станет результатом 3D-печати. Оно вклю- 


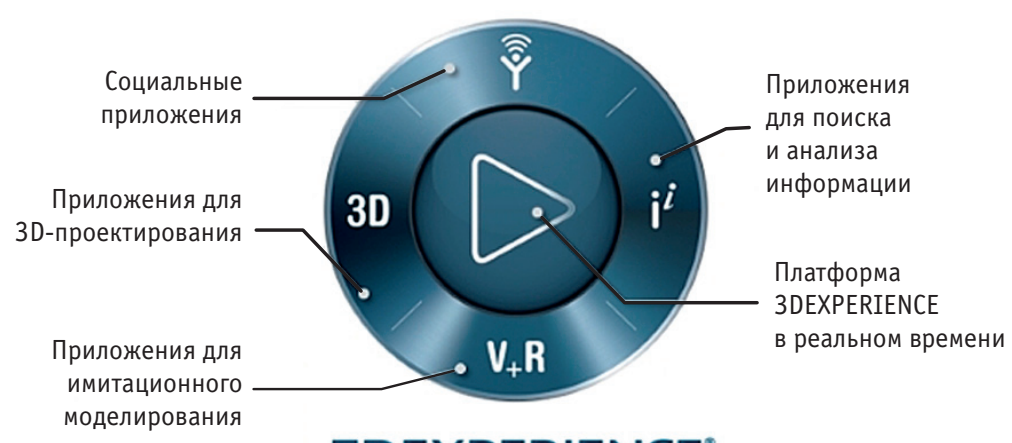

3DEXPERIENCE

управления инженерными данными и документооборотом T-FLEX DOCs закрывает все основные задачи по организации единого информационного пространства предприятия и в полной мере реализует концепцию цифрового макета изделия». Расширенные функции T-FLEX PLM+ позволяют осуществлять управление проектами и планировать ресурсы, вести полноценный организационно-распорядительный документооборот, контролировать взаимоотношения с клиентами и многое другое. «Для мелкосерийного производства часто бывает достаточно создания ядра из современной ERP-системы

Puc. 3. Dassault Systèmes 3DEXPERIENCE - операционная система цифрового предприятия

чает специальную математику, описывающую, например, изменение химических свойств порошка при печати металлом. Параметры этой химической модели напрямую отражаются на качестве физического прототипа - он может оказаться более или менее прочным или упругим. Другие приложения связаны с моделированием виртуальной реальности, а также оптимизацией различных процессов, например, оптимизацией логистики на уровне MESсистем или наилучшим размещением промышленных роботов.

Обе глобальные платформы - Siemens Teamcenter и Dassault Systèmes 3DEXPERIENCE - обеспечивают доступ к данным на основе ролевых моделей, поддерживают облачный режим работы с платформой и возможность расширения спектра приложений за счет решений третьих фирм.

Необходимо отметить российскую PLMсистему T-FLEX PLM, разработанную компанией «Топ Системы». Она, по утверждению вендора, развивается в сторону «тяжелых» систем и намерена в ближайшем будущем составить серьезную конкуренцию зарубежным системам, рассмотренным выше. У нее есть для этого все основания: собственная CAПP T-FLEX CAD, обязательные PLM-подсистемы (CAM, CAE, CAPP технологическая подготовка производства), PDM и базовый набор программных систем для управления предприятием (ERP, электронный документооборот, CRM, обеспечение метрологической службы и т.д.) (рис. 4).

Как рассказал Игорь Кочан, директор по маркетингу компании «Топ-Системы», в основе комплекса лежит первая отечественная PLM-платформа, построенная на основе единой цифровой модели изделия: «Комплекс программ T-FLEX PLM позволяет организовать единую информационную среду конструкторского и технологического проектирования, подготовки и управления производством, сбытом и послепродажным сопровождением изделия. Система

с развитыми функциями планирования и диспетчеризации, - замечает Константин Емельянов из ФРОНТСТЕП СНГ. - Для наиболее сложного «проектирования под заказ» очень важным является конструкторскотехнологическое ПО, включающее систему управления процессами и данными (PLM, PDM)».

Система T-FLEX PLM включает компоненты для работы с параметрическими 2D- и 3D-моделями, инструменты оформления чертежей, поддержку средств виртуальной реальности (VR) непосредственно в процессе моделирования без выгрузки модели в специальные внешние программы, а также механизмы интеграции с различными CADсистемами: CATIA, Creo, NX, SolidWorks, Inventor, SolidEdge и других. Безусловно, это единственная PLM-система «тяжелого» класса, для которой обеспечено согласование данных цифровой модели изделия с военной приемкой.

Можно ли считать внедрение передовой PLMсистемы фактом перехода в состояние цифрового предприятия? Денис Маринич из Softline дает отрицательный ответ: «Ключевой признак цифрового предприятия - единая среда, которая позволяет управлять всем предприятием. Если хотите, его цифровой прототип. Это должна быть именно некая виртуальная сущность, благодаря которой можно смоделировать процесс изготовления нового продукта и оценить, реально ли его произвести в рамках имеющихся производственных мощностей и с какими издержками, как можно будет скорректировать сам продукт и/или перенастроить производственные процессы, и т.д. И по результатам такого моделирования принимать решение. Пока такой платформы не создано».

Действительно, современное состояние платформенных PLM-систем можно охарактеризовать одним словом, которое было вынесено в заголовок основного панельного доклада на отраслевой конференции COFES 2017: «Сложность». Игроки рынка PLM на этот вызов отвечают дальнейшим уси- 


\section{T. FLEX PLM}

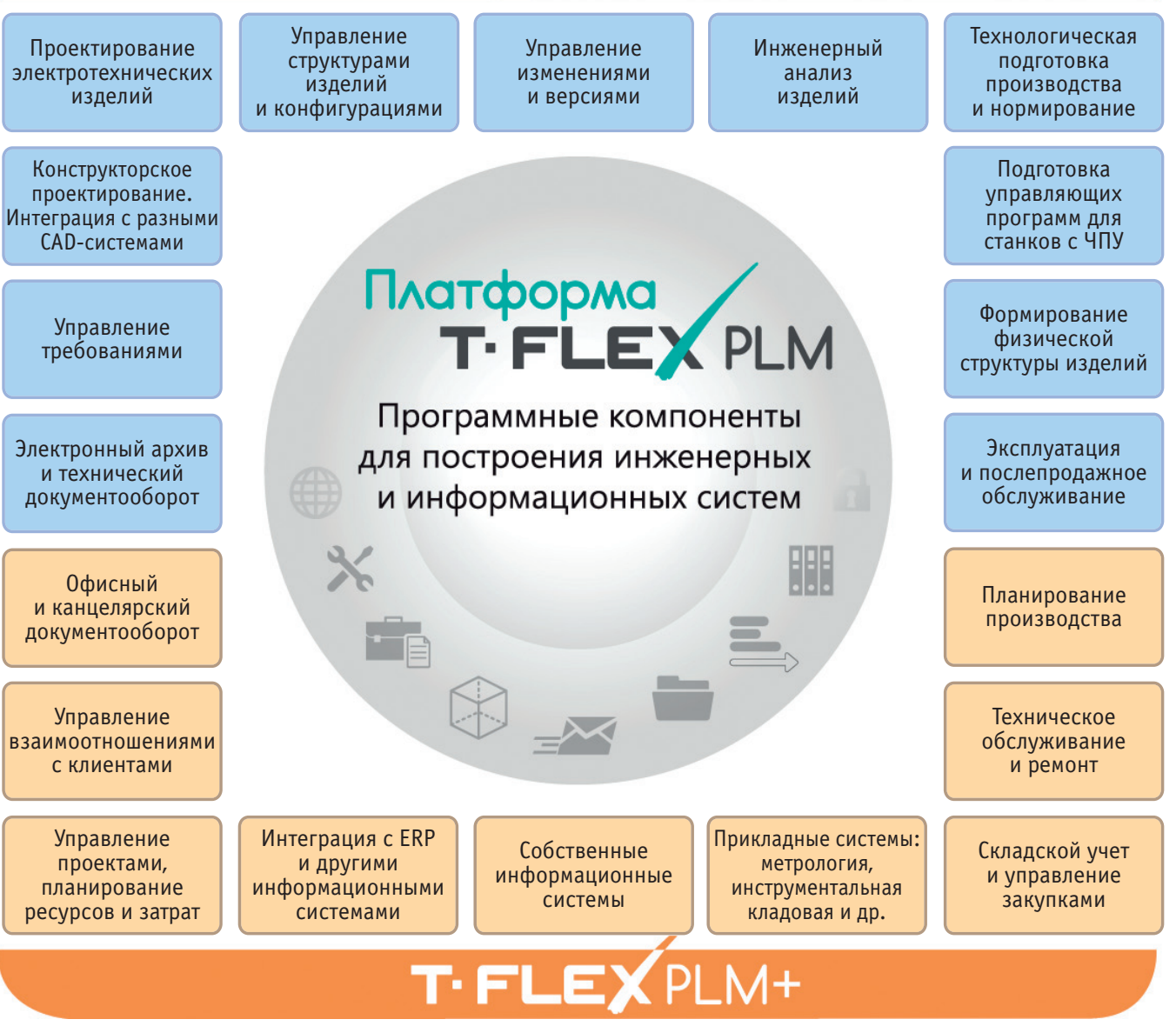

PUc. 4. T-FLEX PLM компании «Топ Системы» - российский вариант «тяжелой» PLM

задача решается путем повышения доступности данных для всех участников жизненного цикла (ЖЦ) изделия, что требует их интеграции в логически единую информационную модель (рис. 5). Поскольку источники данных для единой модели имеют различную природу (чертежи, нормативные документы, результаты анализа и др.) и формируются в автоматическом режиме, поиск ответа на вопросы типа: «Существуют ли необходимые данные?» или «Являются ли эти данные актуальными?» - зачастую оказывается нетривиальной задачей.

Вторая нетривиальная задача обмен данными

лением интеграции и компьютерного инжиниринга, широким использованием математических моделей, переходом к интеллектуальным методам работы с данными.

\section{ВРЕМЯ ИНТЕГРАЦИИ}

Тенденция интеграции в комплексных системах промышленной информатизации не нова: давно известный класс интегрированных САПР включает различные сочетания, такие как CAD/CAM, CAD/ CAE, CAD/CAE/CAM. Рядом с ними развивались компьютерные технологии электронного сопровождения разработки, производства и эксплуатации сложной наукоемкой продукции, так называемые технологии CALS (Continues Acquisition and Life cycle Support). Собственно, одной из ключевых CALSтехнологий является PDM - технология управления данными об изделии.

Основная роль PDM-технологии заключается в том, чтобы сделать информационные процессы максимально прозрачными и управляемыми. Эта с другими системами, в первую очередь, геометрическими моделями и чертежами. Современные САМ-системы поддерживают широкий список импортируемых форматов 2D- и 3D-геометрической информации. К ним относятся как проприетарные (SolidWorks, Parasolid, DWG, NX и др.), требующие наличия соответствующих трансляторов, так и открытые (нейтральные) форматы (STEP, DXF, IGES, IDF, VRML, 3DPDF). Что касается совместимости продуктов глобальных вендоров с отечественными системами, то она обычно реализуется на уровне нейтральных форматов данных. «Пока отечественные САПР системы не будут массово применяться глобально, крупные вендоры не поставят эти форматы в поддержку», - поясняет Алексей Рыжов, генеральный директор Dassault Systèmes в России и СНГ.

К числу наиболее известных PDM-систем относятся, в частности, IPS Search («Интермех»), Teamcenter (Siemens PLM Software), Windchill (PTC), ENOVIA и Solidworks Enterprise PDM (Dassault Systèmes), 1C:PDM (APPIUS), Lotsia PDM PLUS 


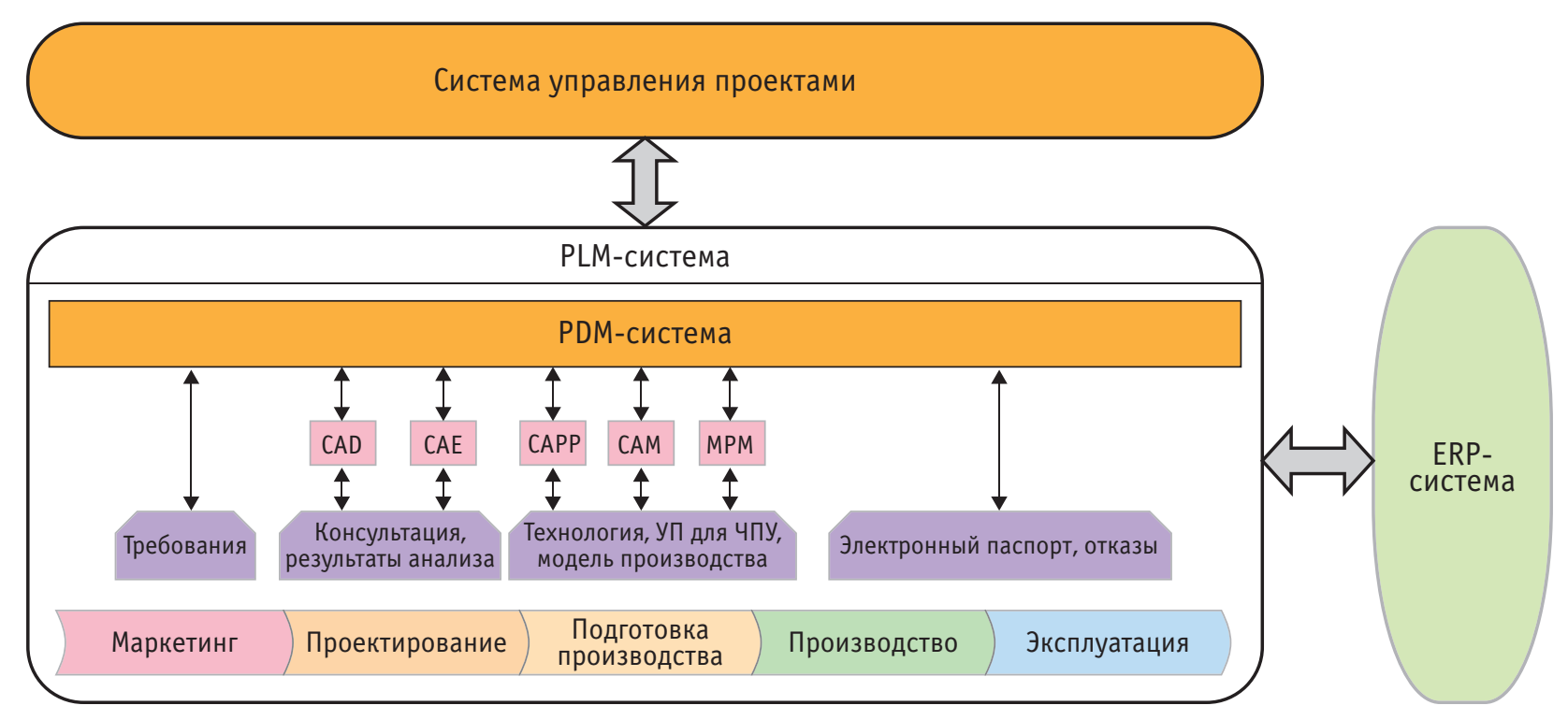

PUc. 5. Интегрированная PLM-система на основе PDM

(«Лоция Софт»), PDM STEP Suite (НИЦ «Прикладная логистика»), ЛОЦМАН:PLM (АСКОН), T-FLEX DOCs («Топ системы»).

\section{СОВЕРШЕНСТВОВАНИЕ ИНТЕГРАЦИОННЫХ МЕХАНИЗМОВ}

Совершенствование интеграционных механизмов в современных PLM-системах идет по нескольким направлениям.

\section{Углубление автоматизации процессов сбора и обработки данных ЖЦ изделия}

«Ключевое отличие цифрового предприятия от традиционной информатизации заключается в степени интегрированности отдельных систем в функциональные подсистемы», - подчеркивает Григорий Абаев из «Би Питрон». Например, система автоматизации проектирования изделия является отдельной системой промышленной автоматизации до тех пор, пока на основе ее данных не будет организована технологическая подготовка производства в некоей единой информационной среде (рис. 6). Яркий пример современной сквозной иерархической интеграции - интеграция систем обработки данных с технологиями промышленного Интернета вещей, которая сейчас активно используется, например, для прогностической аналитики рисков отказов оборудования.

\section{Автоматизация обмена данными между отдельными ИТ-системами предприятия}

«Чем более удобны и гибки будут средства интеграции, тем легче можно будет адаптироваться к новым внедряемым системам и платформам», отмечает Василий Чуранов из компании «Цифра». Перспективным направлением является такая модернизация производственных информационных систем, которая дает возможность встраивать в них наиболее востребованные пользователями управленческие, технологические и аналитические функциональные возможности (рис. 7).

\section{Автоматизация как исключение человеческого фактора}

«Критически важный фактор для качественного управления производством - сбор точных фактических данных о процессах, происходящих в цехах и на складах, - подчеркивает Константин Емельянов из ФРОНТСТЕП СНГ. - Требуется исключение влияния человеческого фактора на качество информации и ее трансляцию в ИТ-систему». Здесь находят применение различные виды электронных маркировок: штрихкодирование, RFID-метки и т.П., а также формирование, выдача, контроль выполнения технологических операций в рамках индивидуальных и бригадных сменно-суточных заданий в электронном виде в автоматическом режиме.

\section{Интеллектуальные средства работы с НСД}

В промышленном производстве используется большое количество прикладных информационных систем различных поставщиков, обладающих собственными базами данных нормативно-справочной информации (НСИ) по материалам и комплектующим, средствам производства и технологического оснащения, контрагентам и контрактам и т.д. Дубли- 
рование баз данных НСИ приводит к повышению стоимости владения ПО, снижению качества информации, необходимости ее синхронизации, риску ошибочных решений на основе недостоверных, разрозненных данных. «Необходимо использовать инфраструктурные решения, обеспечивающие централизованное управление корпоративной нормативно-справочной информацией, - подчеркивает Андрей Андриченко, председатель Совета директоров компании SDI Solution. - Любая прикладная программа, несущая на предприятие собственную базу данных НСИ, обязана синхронизировать свои информационные объекты с эталонными записями в централизованной базе данных НСИ».

Для решения комплекса задач, связанных с централизованным управлением корпоративной справочной информацией, существует специализированный класс систем Master Data Management (MDM), предназначенный для консолидации справочных данных и унификации сервисов по работе с этим типом данных. Ближайшая перспектива MDM - это переход к облачным решениям и стандартизации форматов описания мастер-данных. При выборе MDM-системы необходимо ориентироваться на

ИТ-решения, адаптированные к промышленному производству, которые учитывают специфику работы с инженерными мастер-данными. (Подробнее о MDM-системах для машиностроения рассказывается в статье «Управление корпоративными мастерданными в промышленных холдингах и корпорациях», см.: СТАНКОИНСТРУМЕНТ, 2016, № 4).

Следует отметить, что реализация в MDM объектно-ориентированной модели данных, которая позволяет хранить знания о правилах совместимости материально-технических объектов, позволяет системе осуществлять семантический поиск объектов - в нем принимают участие как параметры искомого объекта, так и правила его совместимости со смежными объектами НСИ. «Применение семантических технологий в системах класса MDM делает их эффективными при работе с инженерными данными, а интеграция MDM

\begin{tabular}{|c|c|c|c|c|c|c|c|c|}
\hline \multicolumn{6}{|c|}{$\begin{array}{c}\text { ERP } \\
\text { (Система планирования и исполнения заказов промышленного производства }\end{array}$} & \multicolumn{3}{|c|}{$\begin{array}{c}\text { CAD/CAMPLM/PDM } \\
\text { (Подготовка производства) }\end{array}$} \\
\hline \multicolumn{6}{|c|}{ I I $\quad$ АИС Диспетчер } & \multicolumn{3}{|c|}{ I } \\
\hline \multirow{2}{*}{ ТОиР } & \multirow{2}{*}{\multicolumn{2}{|c|}{$\begin{array}{c}\text { Аварийный } \\
\text { ремонт }\end{array}$}} & \multirow{2}{*}{$\begin{array}{c}\text { Оперативное } \\
\text { планирование }\end{array}$} & \multirow{2}{*}{$\begin{array}{c}\text { Управление } \\
\text { заданиями }\end{array}$} & \multirow{2}{*}{$\begin{array}{l}\text { Контроль } \\
\text { качества }\end{array}$} & \multicolumn{3}{|c|}{ 0беспечение производства } \\
\hline & & & & & & \begin{tabular}{l|} 
Управление \\
материалом, \\
заготовками
\end{tabular} & $\begin{array}{c}\text { Управление } \\
\text { инструментом }\end{array}$ & $\begin{array}{c}\text { Управление } \\
\text { информацией } \\
\text { (документация, УП) }\end{array}$ \\
\hline \multicolumn{9}{|c|}{ Система мониторинга работы оборудования и производственного персонала } \\
\hline \multicolumn{5}{|c|}{ Мониторинг оборудования } & \multirow{2}{*}{$\begin{array}{c}\text { Мониторинг } \\
\text { рабочих мест } \\
\text { (ЧМИ) }\end{array}$} & \multirow{2}{*}{$\begin{array}{l}\text { Контроль } \\
\text { размеров } \\
\text { изделий }\end{array}$} & \multirow{2}{*}{\begin{tabular}{|c|} 
Мониторинг \\
инструментов, \\
приспособлений
\end{tabular}} & \multirow{2}{*}{$\begin{array}{c}\text { Мониторинг } \\
\text { материалов, } \\
\text { заготовок }\end{array}$} \\
\hline $\begin{array}{l}\text { Состояние } \\
\text { и простои }\end{array}$ & & $\begin{array}{l}\text { ехнические } \\
\text { данные }\end{array}$ & $\begin{array}{c}\text { Технологические } \\
\text { данные }\end{array}$ & $\begin{array}{c}\text { Производственные } \\
\text { данные }\end{array}$ & & & & \\
\hline \multicolumn{3}{|c|}{ Отчеты в реальном времени } & \multicolumn{6}{|c|}{ Аналитика и отчетность } \\
\hline $\begin{array}{c}\text { Линейная диаг } \\
\text { Планировка } \\
\text { Графики сигн }\end{array}$ & & $\begin{array}{l}\text { 0повещения } \\
\text { E-mail, SMS }\end{array}$ & $\begin{array}{c}\text { Статистические } \\
\text { отчеты }\end{array}$ & $\begin{array}{l}\text { Генератор } \\
\text { отчетов }\end{array}$ & $\begin{array}{c}\text { Динамическая } \\
\text { аналитика }\end{array}$ & $\mid \begin{array}{c}\text { Производственные } \\
\text { журналы }\end{array}$ & $\begin{array}{c}\text { Отчеты на } \\
\text { мобильных } \\
\text { устройствах }\end{array}$ & $\begin{array}{c}\text { Отчеты на } \\
\text { мониторах } \\
\text { большого формата }\end{array}$ \\
\hline
\end{tabular}

Puc. 7. Интегрированный комплекс информационных систем на базе системы мониторинга «Диспетчер» 


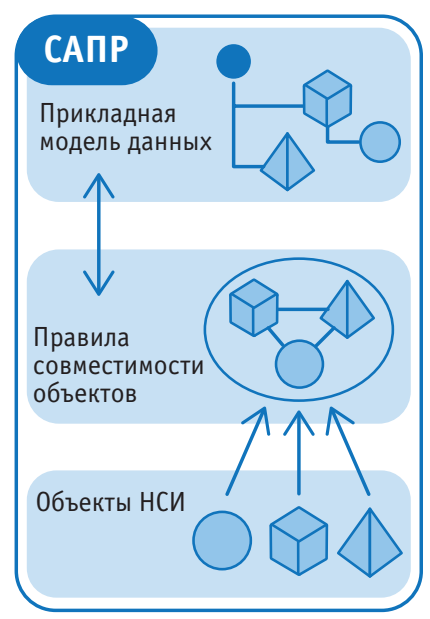

Вчера

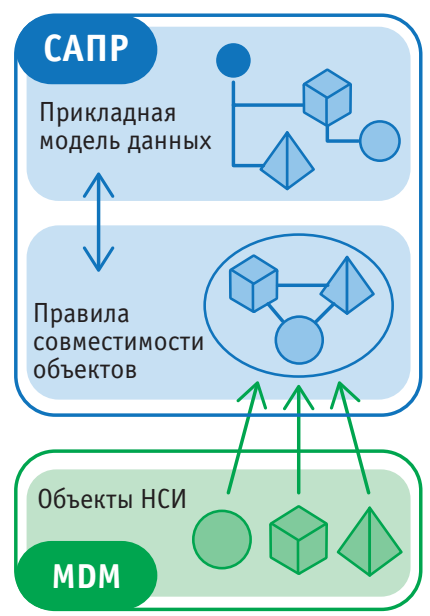

Сегодня

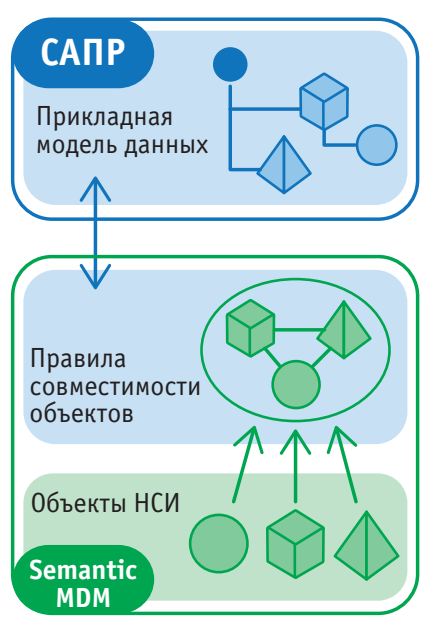

Завтра агентные технологии, реализующие принципы распределенного принятия решений и логические описания агентов прои зв одств енных элементов (например, продуктов, технологических операций, рабочих и станков, материалов и инструментов, а также взаимодействий между ними) (рис. 10).

Puc. 8. САПР - «тонкий клиент» семантической МDM-системы

и САПР позволяет создать новый класс конкурентоспособных интеллектуальных программных комплексов с высоким уровнем автоматизации принятия решений», - отмечает Андрей Андриченко (рис. 8).

\section{Семантические технологии как способ} интеграции данных в гетерогенных системах

Семантические технологии, в числе прочего, дают инструменты для решения болезненных проблем интеграции в гетерогенных средах, включая устаревшие (legacy) системы. В частности, программный продукт InterBridge ГК «НЕОЛАНТ» обеспечивает оперативную трансляцию графических и семантических 2D/3D-данных различных САПР, BIM- и PLM-платформ для работы с единой информационной моделью крупномасштабных технологических объектов. При этом, система управления инженерными данными (СУИД) НЕОСИНТЕЗ, разработанная ГК «НЕОЛАНТ», обеспечивает хранение, доступ, обмен и анализ данных из смежных систем (ERP, АСУ и др.) (рис. 9). Фактически, СУИД НЕОСИНТЕЗ осуществляет управление цифровым активом сложного промышленного объекта на протяжении его жизненного цикла.

\section{ИНТЕЛЛЕКТУАЛЬНЫЕ МЕТОДЫ УПРАВЛЕНИЯ РЕСУРСАМИ ПРЕДПРИЯТИЯ}

Перспективность перехода к интеллектуальным методам управления производственным предприятием сегодня практически не вызывает сомнений. Вопрос - в практической реализации этой идеи. В НПК «Разумные решения» разработано семейство продуктов Smart Factory, предназначенное для интеллектуального управления ресурсами машиностроительных предприятий на стратегическом и оперативном уровне. В основе подхода - мульти-

\section{ИНТЕЛЛЕКТУАЛЬНЫЕ МЕТОДЫ ПОДДЕРЖКИ АВТОМАТИЗИРОВАННОГО ПРОЕКТИРОВАНИЯ}

Как полагает Алексей Боровков из СПбПУ, глобальным вызовам цифровой экономики отвечает тренд автоматизации конструкторской деятельности. Инжиниринговый центр СПбПУ ведет разработку экспертной системы CML-AI, которая должна стать «интеллектуальным помощником» инженера-конструктора в процессе проектирования. Ее задача автоматическая кастомизация и валидация продукта на основе многоуровневой матрицы целевых показателей и ресурсных ограничений, а также специальной базы знаний. На практическом уровне это означает, в частности, что система сама предлагает оптимальную конструкцию под те целевые параметры, которые были заданы конструктором. В целом, данный подход должен охватывать широкий спектр прикладных направлений, формируя систематизированное пространство полезных знаний.

\section{ВРЕМЯ МОДЕЛИРОВАНИЯ}

Математическая модель детали - это центральное звено всей цепочки производственных систем CAD/CAM/CAE/PLM, выступающее в различных ипостасях:

$\rightarrow$ компонент объемной сборки (компоновки) для наглядного представления изделия и изготовления реальных деталей, например, на оборудовании с ЧПУ;

$\rightarrow$ составная часть моделей сопрягаемых с ней деталей и компонент объемной сборки для проверки возможных конфликтов собираемости, взаимных пересечений, соударений и т.п.;

$\rightarrow$ составная часть расчетных алгоритмов и программ, например, для получения массово-инер- 

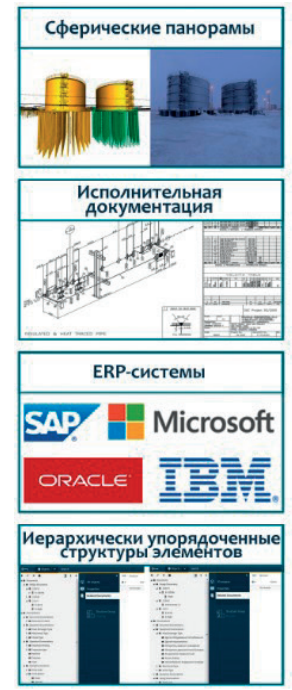

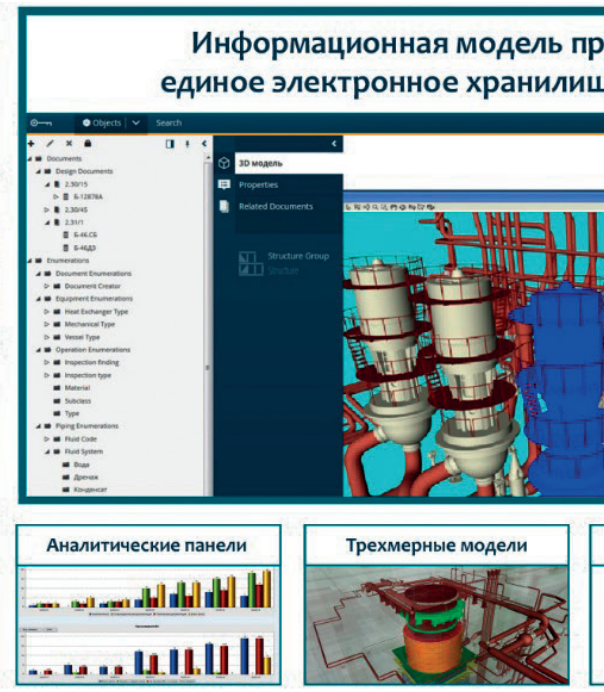

Информационная модель промышленного объекта единое электронное хранилище информации об объекте
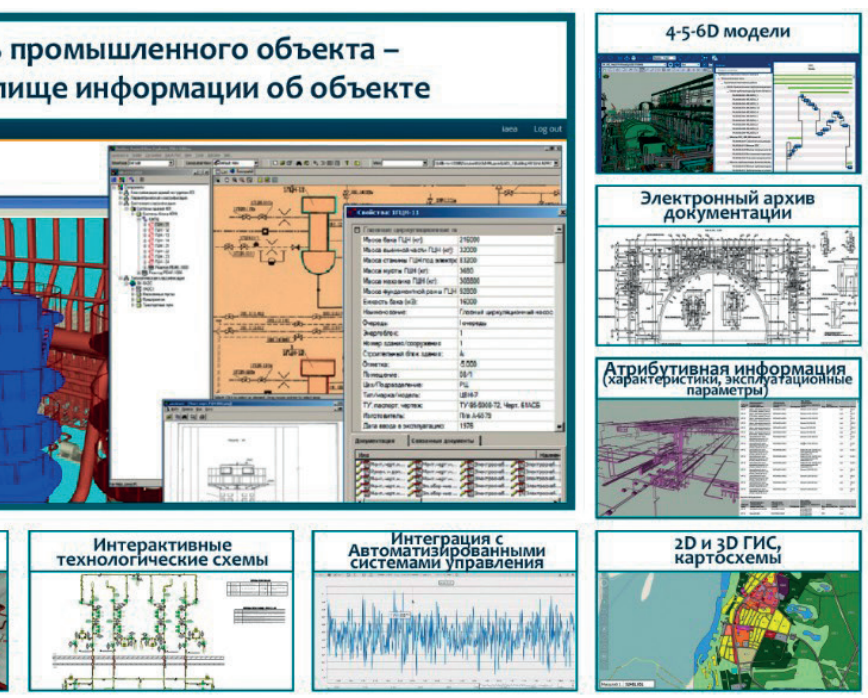

PUс. 9. Система управления инженерными данными НЕОСИНТЕЗ компании «НЕОЛАНТ» осуществляет управление данными единого цифрового актива

ционных характеристик или для анализа прочности методом конечных элементов;

$\rightarrow$ эталон для контроля точности и качества.

Модель изделия - ресурсоемкий элемент, даже по меркам традиционного автоматизированного проектирования. Объем геометрической информации, обрабатываемой на этапе конструкторско-тех-

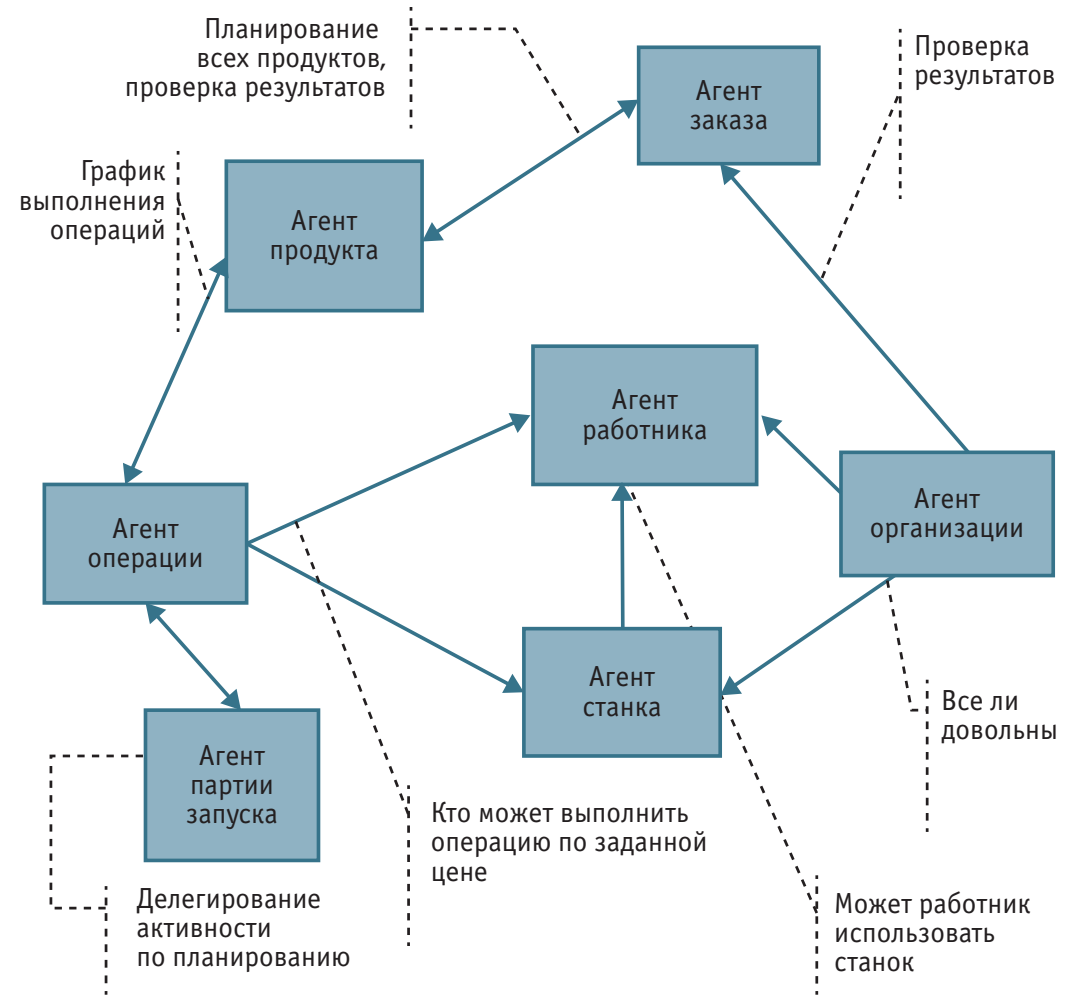

Puc. 10. Основные виды коммуникаций между агентами в мультиагентной системе умной фабрики нологической подготовки производства, возрастает в десятки раз, что обусловливает традиционно высокие требования к производительности используемой вычислительной техники (рис. 11).

Модель является информационной основой всей инфраструктуры современных платформ PLM. «B 3DEXPERIENCE в центре всей логики построения платформы - модель, рассказывает Алексей Рыжов из Dassault Systèmes. - Модель продукции предприятия едина для всех департаментов, предприятий-смежников, поставщиков». В парадигме моделей описывается не только продукция предприятия, но и все производственные процессы, включая методы повышения их эффективности. Действия сотрудников отражаются в моделях в виде описаний их ролей (ролевых моделей). «Очевидно, что применение их на конкретном предприятии подразумевает трансформацию текущих бизнеспроцессов», - подчеркивает Алексей Рыжов. Необходимость трансформации бизнес-процессов, помноженная на трансформацию технологических знаний, вызывает к жизни серьезные проблемы, касающиеся уровня квалификации кадров.

«Следует помнить о том, что даже при работе с программами, нацеленными на решение задач одного класса, качество получаемых результатов может серьезно разниться. Поэтому 


\section{ПУТЬ К ЦИФРОВОМУ ПРЕДПРИЯТИЮ ОБЯЗАТЕЛЬНО ПРОХОДИТ ЧЕРЕЗ РАДИКАЛЬНУЮ РЕОРГАНИЗАЦИЮ БИЗНЕС-ПРОЦЕССОВ?}

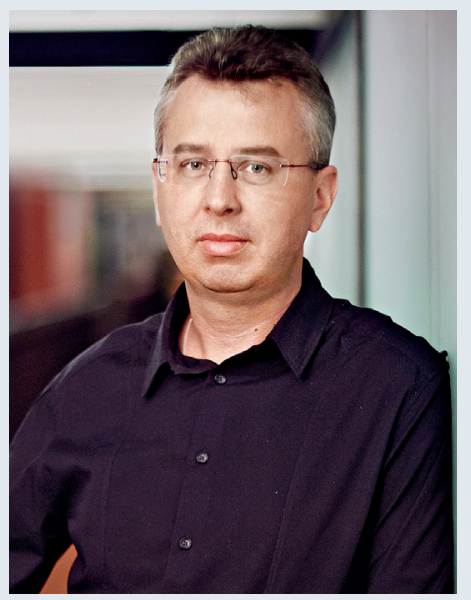

Д. Маринич: «Самое главное - поддержка высшего руководства предприятий. «Топы» должны быть драйверами внедрения таких технологий. У нас был опыт консалтинга, когда мы помогали заказчику избавиться от «зоопарка» систем и перейти на единую платформу. Так вот, к решению этой задачи пришлось привлекать смежников заказчика, чтобы понимать, кто и на каких платформах работает. Если менять все быстро и не обращать внимания на окружающую среду, в которой работает предприятие, есть риск нарушить все связи и парализовать работу завода.

Кстати, необходимость в создании цифрового предприятия есть далеко не у всех. На примере Интернета вещей и бизнес-аналитики на производстве очевидно: далеко не для всех заказчиков имеет экономический смысл собирать информацию с датчиков, станков и прочего оборудования для бесконечной оптимизации и прогнозирования. Цифровое производство - это модно, но это еще и дорого. Для внедрения этого подхода следует понимать экономическую эффективность каждого шага. Думаю, в первую очередь цифровое производство будет востребовано на предприятиях крупносерийного и массового производства».

Г. Абаев: «Зачастую цифровизация бизнес-процессов невозможна без их переосмысления и переориентации на новые технологии: не эффективно закладывать такую же конструкцию моста из стали, какой она была, когда применяли для решения этой задачи дерево. Идея полномасштабной интеграции в единое цифровое предприятие подсказывает, что интеграция отдельных программных решений должна вестись по трем основным направлениям: проекти-

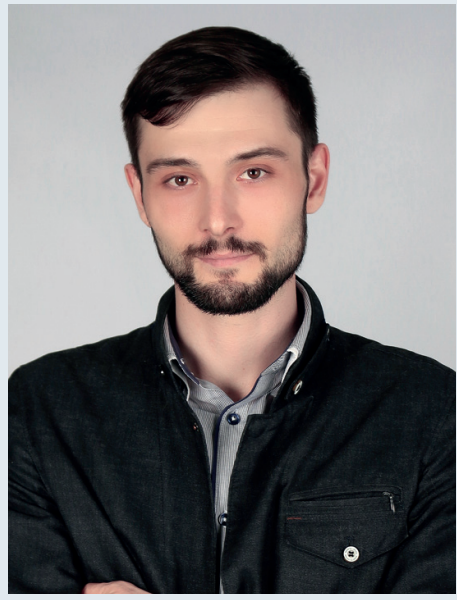

Григорий Абаев, руководитель отдела технической поддержки компании «Би Питрон» рование и управление жизненным циклом изделия (PLM-платформы), производство (ось ERP-MES-SCADA) и кооперация. Такая интеграция отдельных систем в функциональные подсистемы, собственно, и есть «цифровое производство», то есть единая информационная среда функционирования предприятия и его контрагентов при разработке, производстве, эксплуатации и ремонте изделия на основе анализа объективных данных, собираемых на всех этапах ЖЦ».

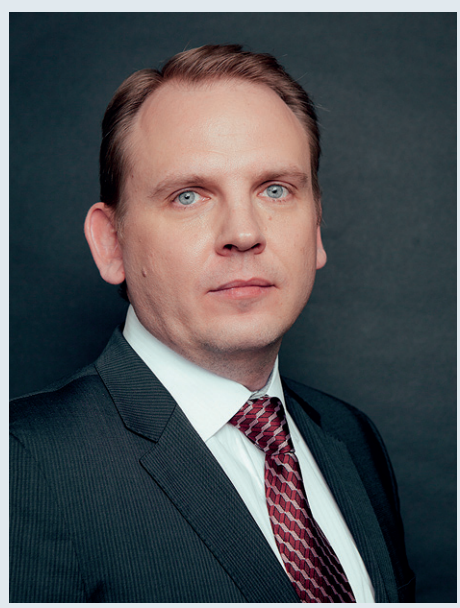

Алексей Рыжов, генеральный директор Dassault Systèmes в России и $\mathrm{CH} \Gamma$

А. Рыжов: «У каждого предприятия есть конкретная бизнес-цель: например, увеличить объем производства, сократить процент брака или сделать так, чтобы сложные изделия проектировались правильно и с первого раза. При этом, у разных предприятий разные приоритеты, в силу того, что «болит» поразному. Нужно говорить в целом про повышение эффективности производства на конкретном предприятии». 


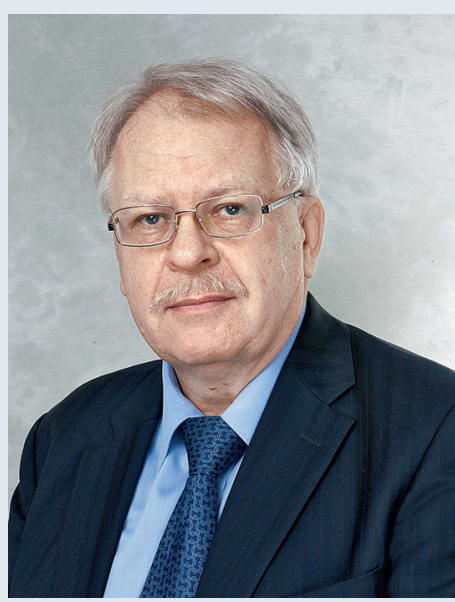

Константин Емельянов, директор по консалтингу ФРОНТСТЕП СНГ

К. Емельянов: «Резкое сокращение времени реакции руководителя на отклонения в планах производства - один из важнейших показателей качества всей системы управления. Должно быть как можно более быстрое и наименее затратное для человека прохождение информации по цепочке: факт, регистрация в системе, информирование руководителя, анализ и введение корректирующих воздействий. Особое внимание должно быть уделено созданию цифровой модели для планирования производства, когда на основе производственного состава изделия, пооперационных норм и набора доступных производственных ресурсов (станки, люди, оснастка, инструмент и т.д. с индивидуальными графиками работы) проводится цифровое моделирование выполнения заказов с назначением свободных ресурсов на задания (планирование) и с последующей оптимизацией выполнения операций внутри ближайших рабочих смен. В результате использования электронного двойника производства должен формироваться реальный график работы станка, участка, цеха, завода». ключевое значение имеет экспертное знание специалиста и арсенал инструментов, которыми он владеет, чтобы принять решение, какая именно программная система даст наилучший результат в каждом конкретном случае», - размышляет Алексей Боровков. В этой связи Григорий Абаев из «Би Питрон» ставит диагноз нынешнему состоянию «умов»: «Многие предприятия не могут сформулировать требования к моделям и ПО. Их позиция проста: используем то, что доступно, закрываем необходимый минимум задач для поддержки бизнес-процессов конструирования, и на том спасибо. Цифровые двойники для многих представляются чем-то недостижимым».

Итог мини-обсуждению подводит Алексей Рыжов: «Вначале руководство должно осознать и принять некие стратегические решения - куда оно хочет двигать свое предприятие. Дальше вступает начальник отдела кадров, который должен четко понимать, что происходит с кадрами: как мы их меняем, как мы их учим, как мы выстраиваем новый тип сотрудников,

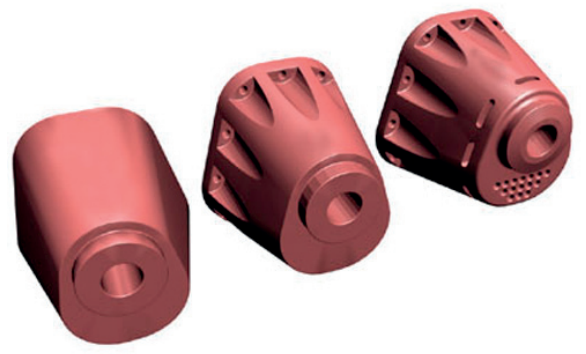

Puc. 11. Степени детализации модели если реально поставлена задача трансформирования предприятия». Денис Маринич из ГК Softline добавляет: «Важно начать выращивать свои кадры. И этим надо заниматься на предприятии: должен быть развитый институт наставничества, организованная система передачи опыта, синергия способностей молодых людей быстро осваивать новые программные и аппаратные решения и опыт ветеранов».

\section{АВТОМАТИЗАЦИЯ ПРОЦЕССОВ СОЗДАНИЯ МОДЕЛЕЙ}

Центральная роль компьютерных моделей на цифровом предприятии обусловливает еще один технологический вызов - автоматизацию самого процесса создания/трансформации моделей. В Центре компьютерного инжиниринга CompMechLab СПбПУ разработана система управления деятельностью в области компьютерного инжиниринга CML-Bench. Она относится к классу SPDM (Simulation and Process Data Management - управление данными и компьютерным моделированием), то есть позволяет автоматизировать выполнение инженерных расчетов, структурировать все расчетные модели и варианты, упростить работу с базой данных расчетных моделей, результатов вычислений и расчетных вариантов, улучшить возможность представления и сравнения результатов инженерных работ (рис. 12).

Систему CML-Bench можно рассматривать в виде платформы междисциплинарной интеграции. К ней присоединяются традиционные системы САПР, в которых формируются геометрические модели изделий. Эта модель погружается в среду CML-Bench, где также размещаются другие модели, охватываю- 


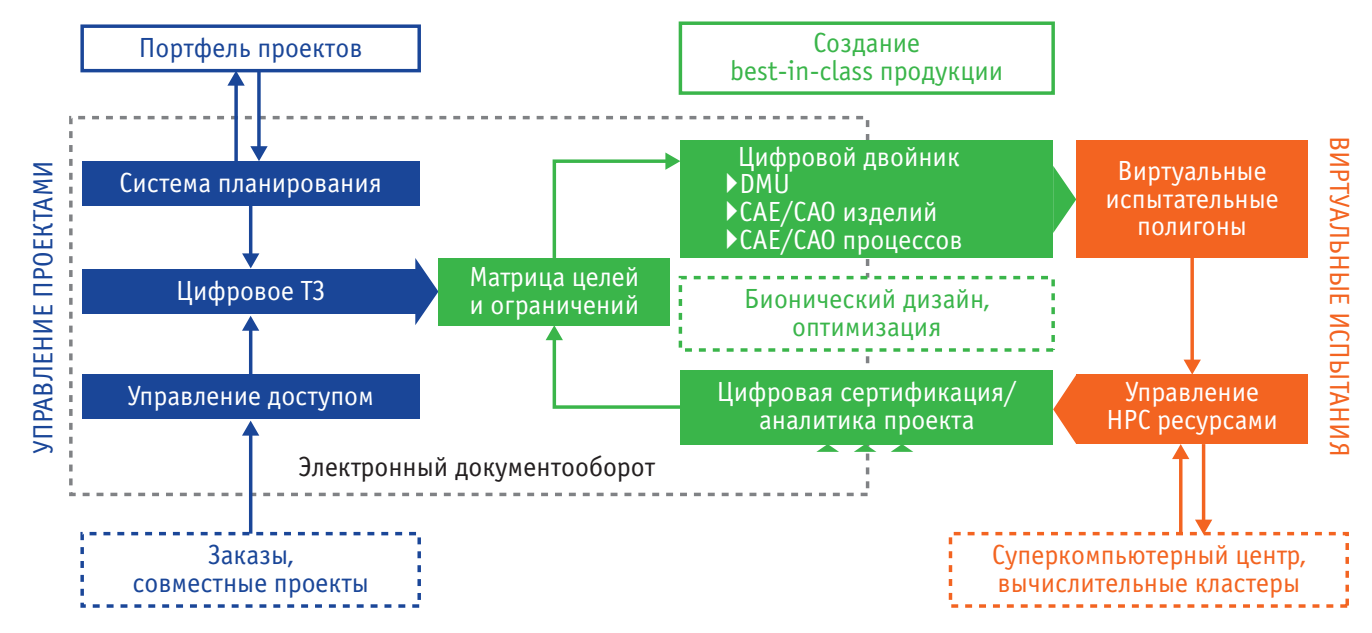

КРОСС-ОТРАСЛЕВАЯ

МУЛЬТИДИСЦИПЛИНАРНАЯ

ЦИФРОВАЯ ПЛАТФОРМА

Управление данными/знаниями/компетенциями/проектами/изменениями/вызовами

Puc. 12. Создание «цифрового двойника» проектируемого объекта в системе CompMechLab сПбПу

щие все этапы ЖЦ изделия, включая сетевые взаимодействия с другими предприятиями и виртуальные испытания. Таким образом, с помощью платформы CML-Bench реализуется идея создания полного цифрового двойника промышленного предприятия.

Цифровая платформа CML-Bench позиционируется разработчиками как универсальный инструмент для создания цифровых фабрик в различных отраслях промышленности: она обеспечивает управление проектами, связанными с цифровым проектированием, моделированием, виртуальными испытаниями и подготовкой конструкторской документации. Она прошла апробацию в отрасли автомобилестроения. В частности, в рамках проекта «Кортеж» (головной исполнитель ФГУП «НАМИ») в части создания единой модульной платформы использовались инструменты цифрового проектирования на основе моделирования, разработанные в инжиниринговом центре СПбПУ. На краш-тестах, проводившихся в Германии и в России, новые автомобили получили максимальные пять «звезд» по шкале безопасности. Для этих целей в центре CompMechLab была проведена цифровая сертификация в соответствии с мировыми требованиями. «Высший балл был получен с первой попытки, что говорит о высоком качестве разработанных моделей», - отмечает Алексей Боровков.

Похоже, что время тотального господства универсальных и громоздких вендорских платформ подходит к концу. Парадигма цифровых предприятий требует новых подходов. «Решение данной задачи требует объединения огромного количества технологий.
Нет такой компании, которая концентрировала бы все компетенции, связанные с цифровым предприятием», - считает Виталий Кононов из ГК «НЕОЛАНТ». Григорий Абаев из «Би Питрон» пытается предположить, как могут выглядеть организационные структуры цифровых предприятий: «Создавать единые информационные платформы на уровне головных о р г а н и з ци й, в о в л е к а ю щ и х в кооперацию более мелких поставщиков, и в рамках конкретного проекта предоставлять доступ всем лицам, задействованным в разработке».

Индустрия экспериментирует с независимыми инжиниринговыми центрами. В качестве рисков такого подхода Константин Емельянов из ФРОНТСТЕП СНГ отмечает сложности документального обеспечения: «Ахиллесова пята независимых инжиниринговых центров, на мой взгляд, - это необходимость учитывать при конструкторско-технологической подготовке производства особенности тех предприятий, где будут производиться будущие изделия. В недалеком прошлом эти задачи решались в практической деятельности серийных КБ и технологических филиалов. В сетевой модели этот вопрос пока не решен».

Суть происходящего хорошо отражает девиз апрельской конференции COFES 2018: «Human-Aided Design: Changing the Relationship between Our Tools and Us». Он говорит, что сегодня в фокусе профессиональных дискуссий об автоматизированном проектировании - вопрос изменения отношений между нашими инструментами и нами.

Редакция журнала «СТАНКОИНСТРУМЕНТ» благодарит все компании и всех экспертов, принявших участие в осмыслении феномена цифрового предприятия с точки зрения применяемого программного обеспечения.

ПОКАТАЕВА Елена -

обозреватель жУрнала «СТАНКОИНСТРУМЕНТ》 